\title{
Total Preperitoneal Robot-Assisted Kidney Transplantation
}

\author{
Clement Michiels, Jean Rouffilange, MD, Vincent Comat, MD, Adeline Guillaume, MD, \\ Samuel Lagabrielle, MD, Henri Bensadoun, MD, PhD, Gregoire Capon, MD, \\ Jean-Marie Ferrière, MD, PhD, Jean-Christophe Bernhard, MD, PhD, and Gregoire Robert, MD, PhD
}

\begin{abstract}
To date, kidney transplant recipients have always undergone open surgery. The type and length of the wound vary, but most commonly, a modified Gibson's incision is made in the lower abdomen for the transplantation. Risk factors for wound complications are well defined in general surgery literature. The laparoscopic kidney transplantation (LKT) technique has been developed recently, and several teams have published studies on the intraperitoneal technique. In this case report, we present our technique of total preperitoneal LKT using the Da Vinci robotic surgical system.
\end{abstract}

Keywords: robotic, kidney transplantation, preperitoneum, surgical technique

\section{Introduction and Background}

$\mathbf{M}$ INIMALLY INVASIVE PROCEDURES have gained widespread acceptance in recent years. In the field of transplantation, laparoscopic living donor nephrectomy requiring a short $(6-10 \mathrm{~cm})$ incision is now considered the optimal procedure. Since the first experience of minimally invasive kidney transplantation, ${ }^{1}$ several teams have published reports on renal transplants performed by robot-assisted transperitoneal laparoscopy. ${ }^{2}$ During transperitoneal approach a large flap of peritoneum is dissected to perform vascular and urinary anastomoses. At the end of the procedure the peritoneum is closed to maintain the renal graft in the iliac fossa and to avoid any twist of the vessels. We report the first totally extraperitoneal laparoscopic kidney transplantation (LKT) using the Da Vinci robotic system.

\section{Procedure of Total Preperitoneum LKT}

After the induction of general anesthesia, a Foley catheter was inserted into the bladder. The patient was positioned dorsal decubitus in the Trendelenburg position at $20^{\circ}$ (Fig. 1).

A 6-cm periumbilical incision was made without incising the peritoneum. The dissection of the preperitoneal space was initiated by the fingers and was progressively enlarged until the iliac vessels. Three robotic ports and two ports for the assistant were inserted once the space was large enough to position trocars under digital control.

The Alexis laparoscopic system with Kii Fios first entry (Applied Medical) was inserted through the 6-cm midline incision once all the trocars were inserted. The robotassisted laparoscopy then began using three 8-mm robotic ports and 11- and 5-mm ports for the assistant as shown in Figure 2.

At this point, the Da Vinci surgical system was docked into position (from the same side as the transplantation), and the robotic arms were attached to the robotic trocars (Fig. 3).

The vessels were dissected using the robot-assisted laparoscopic instruments, with a lymph-node dissection and vessel exposure allowing for the vascular anastomoses. Appropriate hemostasis and lymphostasis were obtained using bipolar forceps, scissors, and metal clips.

The graft was prepared on the back table. To minimize warm ischemia, it was inserted into a laparoscopic plastic bag with crushed ice (Fig. 4).

The Alexis laparoscopic system allows to insert the kidney and to put the optical trocar with the robotic camera arm docked back in place in seconds.

Small holes in the plastic bag were managed to allow simple exposure of the vessels and of the ureter. Stitches were

Department of Urology, Pellegrin Hospital, Bordeaux University Hospital, Bordeaux, France.

(C) Clement Michiels et al. 2017; Published by Mary Ann Liebert, Inc. This is an Open Access article distributed under the terms of the Creative Commons Attribution License, which permits unrestricted use, distribution, and reproduction in any medium, provided the original work is properly cited. 
FIG. 1. Patient positioning.

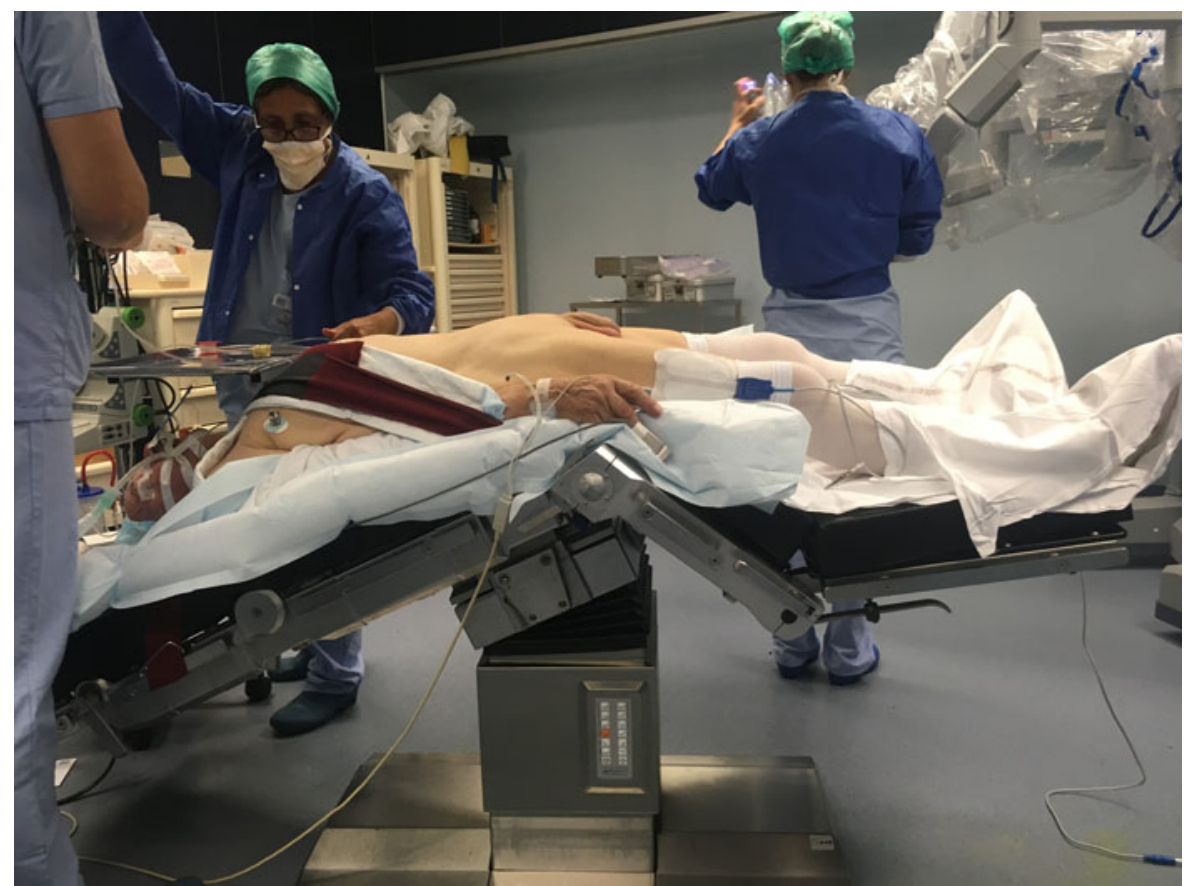

also prepositioned on the vessels to save warm ischemia time.

The graft was brought into the operative field through the midline incision and maintained in good position by the fourth arm. The anastomosis of the renal vein was performed end-to-side of the right external iliac vein with a 6-0 GoreTex running suture.

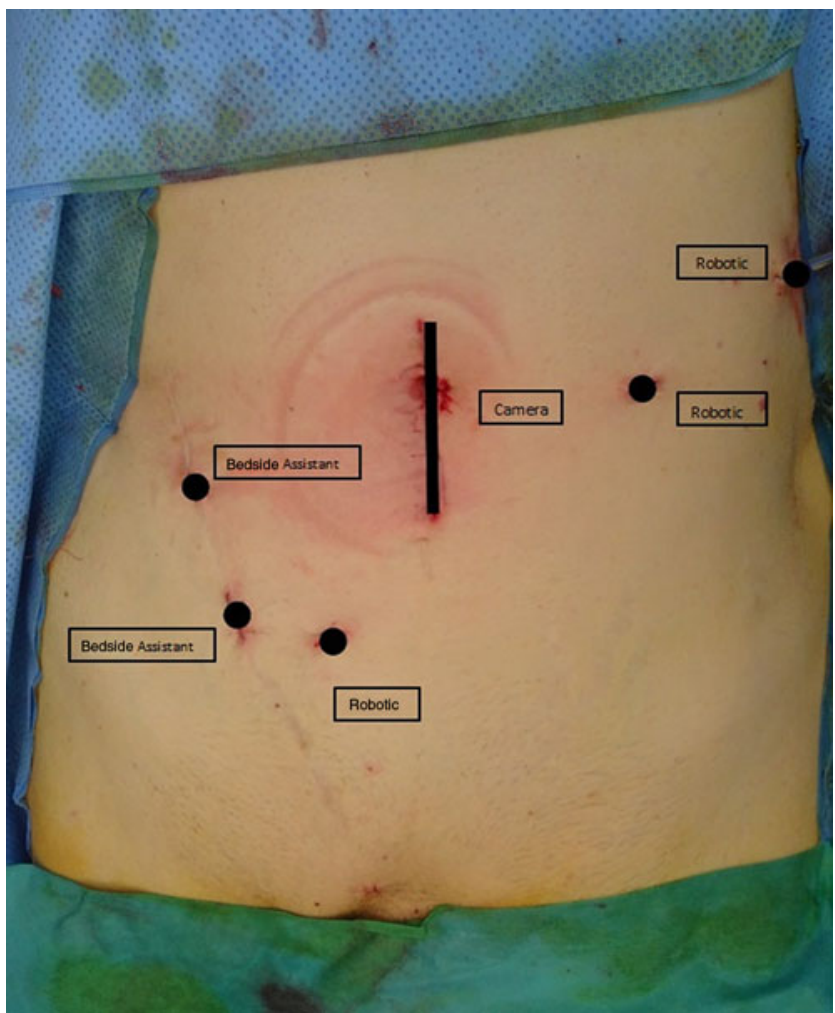

FIG. 2. Positioning of trocars.
The arterial anastomosis was performed end-to-side of the right external iliac artery with a 6-0 Gore-Tex running suture.

The kidney was unclamped, and reperfusion of the parenchyma was observed. Hemostasis was completed when significant bleeding was observed.

Urinary anastomosis was performed using a Lich-Gregoir technique. The bladder was filled with $200 \mathrm{~mL}$ of saline, and

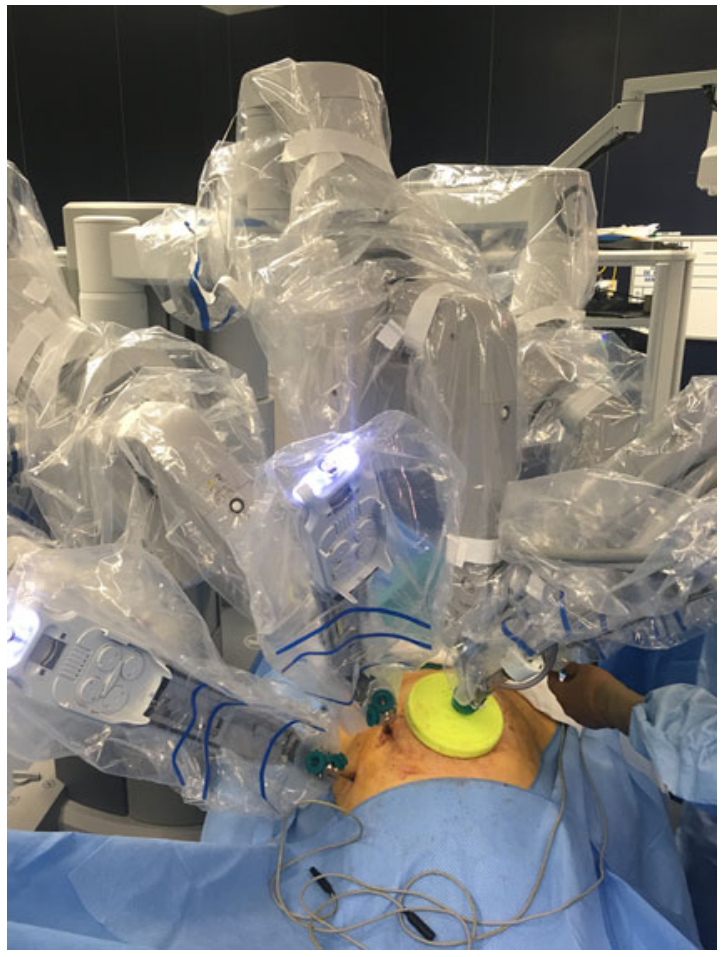

FIG. 3. Robot docked, with bedside assistant opposite side. (Left kidney transplantation.) 


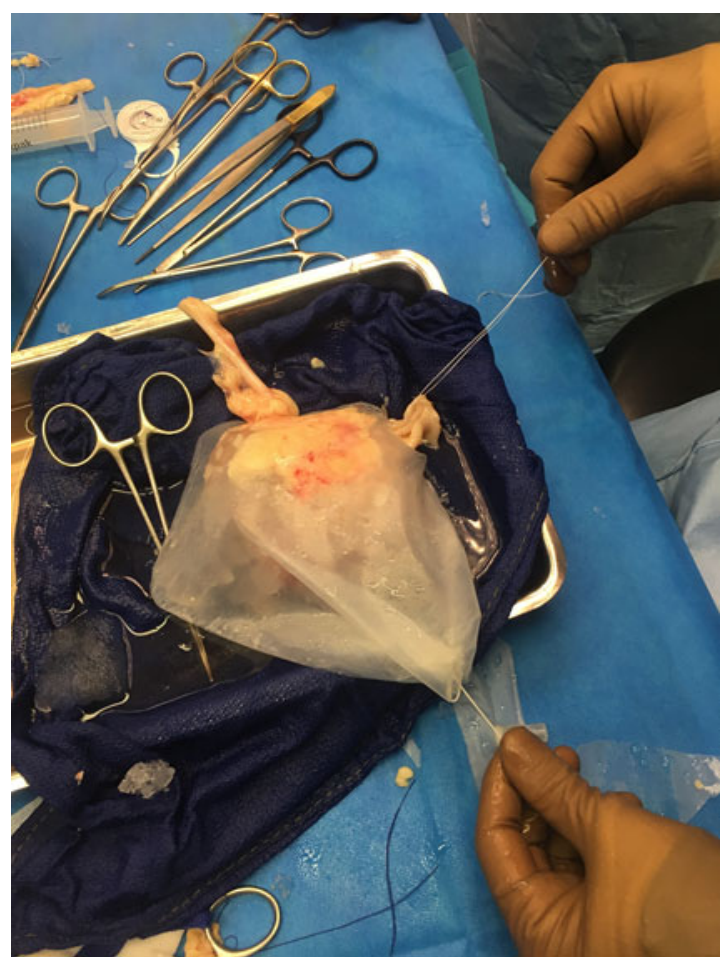

FIG. 4. Cooling laparoscopic bag with prepared kidney graft installed.

the muscular layers were dissected to expose the mucosa. The anastomosis was performed with a running suture of 5-0 PDS, and a Double-J stent was inserted halfway. The outer muscular layers of the bladder were sutured over the distal part of the ureter.

A 9-mm surgical drain was inserted through one of the trocar incisions. The trocars were removed under visual control. The midline incision of the aponeurosis was sutured with Vicryl 2. The cutaneous incisions were sutured with fast-resorption Vicryl 4-0.

\section{Outcomes}

The postoperative sequences were usual with the resumption of function on day 7 . The patient was hospitalized for 10 days. Postoperative pain was managed with perioperative injection of the incisions with Ropivacaine $(20 \mathrm{cc}$ of $7.5 \mathrm{mg} / \mathrm{mL}$ ), as well as intravenous paracetamol and tramadol for the first 24 hours, then switched orally. The postoperative day 1 and day 5 ultrasound and Doppler examinations did not show any anomaly.

Postoperative day 7 creatinine level was at $2.64 \mathrm{mg} / \mathrm{dL}$ and at $2.36 \mathrm{mg} / \mathrm{dL}$ on postoperative day 10 . The patient did not require postoperative hemodialysis.

\section{Discussion}

For kidney transplantation a large iliac incision is usually required to insert the graft and to perform the anastomoses. Incisions for kidney transplantation are usually $16-20 \mathrm{~cm}$ leading to significant postoperative pain and to some delayed wall complications well described in the literature. ${ }^{3}$ The feasibility and safety of kidney transplantation using robotic approach have already been reported. However, smaller incisions require excessive retraction of the wound, which causes bruising and trauma to the muscles. Furthermore, in patients with a high body mass index, it is difficult to access the pelvic vessels despite maximum retraction, which makes dissection and vascular anastomoses cumbersome. Prolonged and excessive retraction causing damage to the femoral nerve, injury to the muscles, and drying of the wound may cause wound-related problems, especially in obese recipients. In fact, LKT would be a boon to patients with high body mass index because it requires only an incision to insert and place the renal graft. Additional port placement may be required, but it is still a better bargain than a larger incision for open kidney transplantation. Nevertheless, the preperitoneal laparoscopic approach is a technically feasible option as we show in this article. Our description of robot-assisted LKT is not an innovation as this intervention is already performed in several university hospitals worldwide. To us, the total preperitoneal laparoscopic access was a very interesting option for exposure and kidney transplant, as it is described and used for traditional kidney transplantation and retroperitoneoscopic donor nephrectomy. ${ }^{4}$ It facilitates access to the vessels, with a more comfortable exposure, without having to retract the bowels and open a flap of peritoneum.

In the end, the kidney isn't free in the peritoneal cavity, in contact with the bowels, but it is confined in this preperitoneal space, placed more securely. Furthermore, in the scope of mini-invasive surgery and enhanced recovery, preperitoneal LKT should reduce postoperative ileus and bowel surgical complications. It reproduces the open kidney transplantation setting which is preferably done in the first place extraperitoneally for an easier access to the iliac vessels without exposure problems due to the intestinal loops and thus, as written above, no bowel complications whatsoever. The kidney is placed in a more adequate manner without contact with the bowels and without excessive mobility which could compromise the transplant vasculature when the kidney is placed inside the peritoneal space.

Our cooling device allows the cooled area to be confined to the kidney and around the kidney without interfering with the anastomoses as the ureter and the vascular pedicle are, through the bag's holes, easily manipulated for exposure and suturing, all while the kidney stays in contact with shaved ice during the whole warm ischemia time. Other cooling systems, like rinsing the kidney with cold saline during warm ischemia time, may be less efficient and cause other problems favoring surgical site infections or bowel complications. It is cheap and simple to make, a practical homemade solution.

\section{Conclusion}

Our cooling device is a simple inexpensive system that works. Research has been done to develop cooling systems and evaluate their quantitative efficiency to keep the graft cold enough during warm ischemia. This technique, however, requires robotic surgery and transplantation expertise; it also deserves an analysis of a series of patients to study the results and compare them to open transplantation. 


\section{Authors' Contribution}

All authors substantially participated in the conception, design, and execution of the study, data analysis and interpretation, and drafting and editing of the article.

\section{Disclosure Statement}

No competing financial interests exist.

\section{References}

1. Oyen O, Scholz T, Hartmann A, Pfeffer P. Minimally invasive laparoscopic transplantation: The first experience. Transplant Proc 2006;38:2798-2802.

2. Modi P, Rizvi J, Pal B, et al. Laparoscopic kidney transplantation: An initial experience. Am J Transplant 2011;11: 1320-1324.

3. Gislason $\mathrm{H}$, Soreide O, Viste A. Wound complications after major gastrointestinal operations. The surgeon as a risk factor. Dig Surg 1999;16:512.

4. Klop KWJ, Kok NFM, Dols LFC, Dor FJMF, Tran KTC, Terkivatan T, Weimar W, Ijzermans JNM. Can right-sided hand-assisted retroperitoneoscopic donor nephrectomy be advocated above standard laparoscopic donor nephrectomy: A randomized pilot study. Transpl Int 2014;27:162-169.
Address correspondence to: Grégoire Robert, $M D, P h D$ Department of Urology Pellegrin Hospital Bordeaux University Hospital Place Amelie Raba Leon 33000 Bordeaux

France

E-mail: gregoire.robert@chu-bordeaux.fr

Abbreviation Used
LKT $=$ laparoscopic kidney transplantation

Cite this article as: Michiels $\mathrm{C}$, Rouffilange $\mathrm{J}$, Comat V, Guillaume A, Lagabrielle S, Bensadoun H, Capon G, Ferrière J-M, Bernhard J-C, Robert G (2017) Total Preperitoneal Robot-Assisted Kidney Transplantation, Journal of Endourology Case Reports 3:1, 169-172, DOI: 10.1089/cren.2017.0098. 